\title{
Combination Irbesartan/Amlodipine versus Irbesartan/Cilnidipine for Attenuation of Albuminuria in Rats with Streptozotocin-Induced Diabetic Nephropathy
}

\author{
Minoru Satoh*, Yuko Nishi, Hiroyuki Kadoya, Seiji Itano, Norio Komai, Tamaki Sasaki and Naoki Kashihara
}

Department of Nephrology and Hypertension, Kawasaki Medical School 577 Matsushima, Kurashiki, Okayama 701-0192, Japan

\begin{abstract}
Background: Excessive urinary albumin excretion is associated with hypertension and diabetic nephropathy. Calcium channel blockers (CCBs) used as antihypertensives suppress such albuminuria with variable efficacy. While hypertension benefits from the addition of angiotensin receptor blockers (ARBs), it is unknown if ARBs alter the effects of CCBs on albuminuria.

Objective: This study compared the efficacy of combined ARB irbesartan with either CCB amlodipine or CCB cilnidipine on albuminuria associated with experimental diabetic nephropathy.

Methods: Male Sprague-Dawley rats with streptozotocin-induced diabetes were treated with a CCB alone (amlodipine $2.0 \mathrm{mg} / \mathrm{kg} / \mathrm{d}$ or cilnidipine $2.0 \mathrm{mg} / \mathrm{kg} / \mathrm{d}$ ), an ARB alone (irbesartan $20.0 \mathrm{mg} / \mathrm{kg} / \mathrm{d}$ ), or combinations. In the acute protocol, changes in glomerular afferent and efferent arteriole diameters were examined by a charge-coupled device video microscope following single doses. In the chronic protocol, urinary albumin excretion, glomerular reactive oxygen species, and endothelial surface layer (ESL) condition were evaluated after 2 weeks of daily treatment.

Results: In the acute protocol, cilnidipine mono therapy caused a greater dilation in glomerular efferent arterioles than amlodipine monotherapy, while combination therapy with irbesartan induced comparable efferent arteriole dilation. In the chronic protocol, cilnidipine mono therapy suppressed albuminuria, reduced glomerular oxidative stress, and protected the glomerular ESL against degeneration to a much greater extent that amlodipine monotherapy. However, addition of irbesartan reduced albumin excretion, oxidative stress, and ESL degeneration to the same extent in both groups.

Conclusions: While cilnidipine is more effective alone, the combinations of irbesartan with cilnidipine or amlodipine are equally effective for reducing albuminuria and other pathological sequela of experimental diabetic nephropathy.
\end{abstract}

Keywords: Angiotensin receptor blocker; Calcium channel blocker; Diabetic nephropathy; Albuminuria

Abbreviation: RAS: Renin angiotensin system; ARB: Angiotensin receptor blocker; CCB: Calcium channel blocker; DN: Diabetic nephropathy; CCDV: Charge-coupled device video; Ccr: Creatinine clearance; WGA: Wheat germ agglutinin; DCF: 2';7'-Dichlorofluorescein; DCFH: 2';7'-Dichlorodihydrofluorescin; PCR: Polymerase chain reaction; SBP: Systolic blood pressure; ESL: Endothelial surface layer

\section{Introduction}

Managing hypertension is important for reducing the risks of cardiovascular and renal diseases $[1,2]$. Renin angiotensin system (RAS) inhibitors are recommended for cardiovascular or chronic kidney disease [3]; however, it can be difficult to achieve the recommended target blood pressure $(<130 / 80 \mathrm{mmHg})$ using angiotensin receptor blocker (ARB) monotherapy [4]. The most effective method to attain good blood pressure control is combination therapy with two or more agents. In particular, combination therapy with ARB and calcium channel blockers (CCBs) is efficacious and safe, and recommended by both the Seventh Report of the Joint National Committee (JNC 8) [5] and the Japanese Society of Hypertension Guidelines for the Management of Hypertension (JSH 2014) [6].

Cilnidipine is a dihydropyridine CCB that acts on both L-type and $\mathrm{N}$-type calcium channels. It has been reported to inhibit sympathetic nerve activity [7] and confers a greater renoprotective effect than L-type-selective CCBs. These L-type CCBs preferentially dilate afferent arterioles, whereas L/N-type CCBs potently dilate both afferent and efferent arterioles [8], and hence the intraglomerular pressure may be reduced by cilnidipine but not by L-type CCB treatment. Indeed, studies have found that cilnidipine is superior to amlodipine, an L-type $\mathrm{CCB}$, for preventing the progression of proteinuria in hypertensive patients undergoing treatment with RAS inhibitors [9].

Pharmacological targeting of the RAS not only reduces blood pressure, but may also provide more direct vascular and organ protection. Clinical studies have demonstrated the efficacy of irbesartan, losartan, telmisartan and valsartan in the management of

*Corresponding author: Minoru Satoh, MD, PhD, Department of Nephrology and Hypertension, Kawasaki Medical School, 577 Matsushima, Kurashiki, Okayama 701-0192, Japan, Tel: +81 (86) 462-1111; Fax: +81 (86) 464-1039; E-mail: msatoh@med.kawasaki-m.ac.jp

Received January 06, 2015; Accepted May 02, 2015; Published May 09, 2015

Citation: Satoh M, Nishi Y, Kadoya H, Itano S, Komai N, et al. (2015) Combination Irbesartan/Amlodipine versus Irbesartan/Cilnidipine for Attenuation of Albuminuria in Rats with Streptozotocin-Induced Diabetic Nephropathy. Pharm Anal Acta 6: 369. doi:10.4172/21532435.1000369

Copyright: $\odot 2015$ Satoh M, et al. This is an open-access article distributed under the terms of the Creative Commons Attribution License, which permits unrestricted use, distribution, and reproduction in any medium, provided the original author and source are credited. 
CKD [10]. Among of ARBs, irbesartan has been shown to be effective in both early and late stage diabetic nephropathy by large-scale randomized clinical study [11]. Compared with valsartan, irbesartan showed the slower decay and longer duration of its antagonistic effects with [12] or without hydrochlorothiazide [13,14]. In addition, irbesartan has been reported to restore sympathetic vasoconstriction in the hypertensive patients [15]. Moreover, irbesartan has peroxisome proliferator-activated receptor agonistic effects [16]. So, irbesartan may be beneficial for treatment of diabetic patients compared with other ARBs.

We hypothesized that combination therapy with irbesartan exceed the superiority of cilnidipine to amlodipine on diabetic nephropathy (DN) treatment. This study compared the efficacy of irbesartan plus amlodipine versus irbesartan plus cilnidipine on glomerular microcirculation and renal function in rats with streptozotocininduced DN.

\section{Methods}

\section{Acute administration protocol}

All experimental protocols were approved by the Animal Research Committee of Kawasaki Medical School (Approval No.12-039), conducted according to the "Guide for the Care and Use of Laboratory Animals" of Kawasaki Medical School (Kurashiki, Japan), and conformed to the "Recommendations on the Establishment of Animal Experimental Guidelines" approved at the $80^{\text {th }}$ General Assembly of the Japanese Science Council in 1980. Twenty-four male Sprague-Dawley rats age 6-7 weeks and weighing 140-160 g were obtained from Charles River Japan (Kanagawa, Japan). The acute administration protocol is shown in Figure 1A. Briefly, diabetes was induced by a single injection of streptozotocin $(65 \mathrm{mg} / \mathrm{kg}$ body weight; Sigma-Aldrich Japan, Tokyo, Japan) diluted in citrate buffer ( $\mathrm{pH}$ 4.5) into the tail vein [17]. Six weeks after the injection, diabetic rats (as determined by fasting serum glucose $>300 \mathrm{mg} / \mathrm{dL}$ ) were randomly divided into 6 equal groups of 4 : (1) diabetic control group (DM, no treatment); (2) amlodipine group (Amlo, single administration of $2.0 \mathrm{mg} / \mathrm{kg}$ ); (3) cilnidipine group (Cilni, single administration of $2.0 \mathrm{mg} / \mathrm{kg}$ ); (4) irbesartan group (Irbe, single administration of $20.0 \mathrm{mg} / \mathrm{kg}$ ); (5) Irbe + Amlo group (single doses of irbesartan $20.0 \mathrm{mg} / \mathrm{kg}$ and amlodipine $2.0 \mathrm{mg} / \mathrm{kg}$ ); and (6) Irbe + Cilni group (single doses of irbesartan $20.0 \mathrm{mg} / \mathrm{kg}$ and cilnidipine $2.0 \mathrm{mg} / \mathrm{kg}$ ). Sprague-Dawley rats injected with an equal volume of citrate buffer served as non-diabetic controls (Cont). Amlodipine and Irbesartan were provided by Sumitomo Dainippon Pharma Co. (Osaka, Japan). Cilnidipine was purchased from Sigma-Aldrich Japan.

Under anesthesia with sevoflurane, oxygen, and nitrous oxide, a stomach tube was inserted into the duodenum for oral gavage administration of drugs. Blood pressure was measured by the tail cuff method (BP-98A; Softron, Tokyo, Japan). Glomeruli in the left kidney were examined using a needle-probe charge-coupled device video (CCDV) microscope as previously reported $[18,19]$. To obtain images of glomeruli, the tip of the CCDV probe was inserted into the kidney through a small $0.5-1 \mathrm{~mm}$ incision as previously described [18]. Imaging of glomeruli was initiated prior to drug administration. Imaging was started upon drug administration and continued for $30 \mathrm{~min}$ thereafter. The diameters of the afferent and efferent arterioles prior to administration and at maximum dilation following administration were measured using ImageJ software (http://rsbweb. nih.gov/ij/; accessed 1 November 2014). Results are expressed as the percent change in diameter compared to non-diabetic controls (defined as 100\%). In addition to glomerular imaging, the right ureter
A Acute administration protocol Week 0: DM induction by STZ Week 6: 30 min recording by CCDV Cont group Non-DM control DM groups

DM: No treatment

Amlo: Amlodipine $(2.0 \mathrm{mg} / \mathrm{kg})$

Cilni: Cilnidipine $(2.0 \mathrm{mg} / \mathrm{kg})$

Irbe: Irbesartan $(20.0 \mathrm{mg} / \mathrm{kg})$

Irbe + Amlo: Irbesartan plus amlodipine

Irbe + Cilni: Irbesartan plus cilnidipine

B Chronic administration protocol

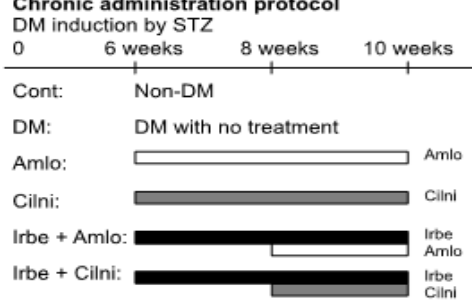

$\square$ Amlo: Amlodipine $(2.0 \mathrm{mg} / \mathrm{kg} / \mathrm{d})$

Irbe: Irbesartan $(20.0 \mathrm{mg} / \mathrm{kg} / \mathrm{d})$

Figure 1: Experimental protocol. (A)Acute drug administration protocol, $n=4$ rats per treatment group. (B) Chronic drug administration protocol, $n=7$ rats per treatment group.

was cannulated and urine collected for volume measurement and urine creatinine determination. At the end of the experiment, blood was collected for measurement of creatinine clearance (Ccr).

\section{Chronic administration protocol}

The chronic administration protocol is shown in Figure 1B. Male Sprague-Dawley rats age 6-7 weeks and weighing 140-155 g were obtained from Charles River Japan. Diabetes was induced using the same protocol as in the acute administration protocol. Age-matched non-diabetic control rats (Cont, $\mathrm{n}=6$ ) were injected with an equal volume of citrate buffer. Six weeks after the induction of diabetes, rats were randomly divided into 4 groups: (1) DM group ( $n=7$, no other treatment), (2) Amlo group ( $\mathrm{n}=7$, amlodipine $2.0 \mathrm{mg} / \mathrm{kg} / \mathrm{d}$ ), (3) Cilni group ( $\mathrm{n}=7$, cilnidipine $2.0 \mathrm{mg} / \mathrm{kg} / \mathrm{d})$, and (4) Irbe group $(\mathrm{n}=14$, irbesartan $20.0 \mathrm{mg} / \mathrm{kg} / \mathrm{d}$ ). After 2 weeks of daily treatment, all rats were placed in metabolic cages to collect a 24 -h urine sample. For an additional 2 weeks, half of the Irbe group was treated with irbesartan plus amlodipine $2.0 \mathrm{mg} / \mathrm{kg} / \mathrm{d}$ (Irbe + Amlo group) while the other half was treated with irbesartan plus cilnidipine $2.0 \mathrm{mg} / \mathrm{kg} / \mathrm{d}$ (Irbe + Cilni group). During the experimental period, body weights were measured weekly. Blood pressure was also measured every week by the tail cuff method (BP-98A; Softron). After collecting a second urine sample, all rats were euthanized and blood was immediately collected in centrifuge tubes. The serum was isolated and assayed for creatinine, blood urea nitrogen, and glucose. Albumin concentrations in the urine samples were measured using an enzyme-linked immunosorbent assay kit (Exocell, Philadelphia, PA) at 2 and 4 weeks after treatment. The left kidney was preserved in $4 \%$ paraformaldehyde and then embedded in paraffin for histochemistry. The right kidney was cut into small pieces, and glomeruli were isolated by mechanical graded sieving for superoxide production assay and extraction of mRNA.

\section{Histological and immunohistochemical staining}

Paraffin-embedded tissue samples were cut into $4-\mu \mathrm{m}$ thick 
Citation: Satoh M, Nishi Y, Kadoya H, Itano S, Komai N, et al. (2015) Combination Irbesartan/Amlodipine versus Irbesartan/Cilnidipine for Attenuation of Albuminuria in Rats with Streptozotocin-Induced Diabetic Nephropathy. Pharm Anal Acta 6: 369. doi:10.4172/21532435.1000369

sections and stained with periodic acid Schiff and tetramethyl rhodamine isothiocyanate-conjugated wheat germ agglutinin (WGA) (Vector Lab, Burlingame, CA) [20]. For immunohistochemical staining, paraffin embedded specimens were rehydrated in phosphate buffered saline and subjected to antigen retrieval in a microwave oven. Antibodies against podocin (Santa Cruz Biotechnology, Santa Cruz, CA) were used as the primary antibodies, and the DAKO EnVision+ system and diaminobenzidine reagent (Dako Japan, Kyoto, Japan) were used for antibody detection. Glomerular volume was evaluated by light microscopy. Volumes of the glomerular tuft were calculated from midsection areas using the maximal planar area method [21]. The WGA and podocin staining scores were determined according to the method described by Macconi et al. [22]. A score was assigned to each individual glomerulus in the tissue section as follows: continuous staining along the glomerular capillary wall (score of 0 ), heterogeneous staining along the glomerular membrane with variable intensity from one region to another within the same glomerulus (score of 0.5 ), and markedly discontinuous patchy staining (score of 1.0). The final score per section was then calculated as the weighted mean: score $=(\mathrm{N} 1 \times 0+$ $\mathrm{N} 2 \times 0.5+\mathrm{N} 3 \times 1) /(\mathrm{N} 1+\mathrm{N} 2+\mathrm{N} 3)$, where $\mathrm{Ni}(\mathrm{i}=1$ to 3$)$ is the number of glomeruli in each category. The scores were assigned in blind fashion. On average, more than 80 glomeruli per section were evaluated. Glomerular volumes and WGA and podocin staining scores were evaluated for at least 20 randomly selected glomeruli from the renal cortex in each rat (a total of 100 glomeruli from 5 rats in each group), and the mean scores were calculated.

\section{Detection of glomerular superoxide}

Glomerular superoxide production was measured by 2',7'-dichlorofluorescein (DCF) staining [23]. Isolated glomeruli from each rats were incubated with RPMI-1640 containing $20 \mu \mathrm{M}$ 2',7'-dichlorodihydrofluorescin (DCFH) diacetate (Molecular Probes, Eugene, OR) for $10 \mathrm{~min}$ and then rinsed with phosphate buffered saline. Fluorescence images were obtained using a confocal laser microscope (TCS SP2 AOBS MP; Leica Microsystems, Tokyo, Japan) at excitation/ emission wavelengths of $485 / 535 \mathrm{~nm}$ for DCF. The fluorescence intensity values from 20 different isolated glomeruli per rats (a total of 100 glomeruli from 5 rats in each group) were calculated by Leica TCSNT software and are presented as mean values.

\section{Quantitative Real-time Polymerase Chain Reaction (PCR)}

RNA isolation and quantitative real-time PCR for heparanase and podocin were performed as previously described [24]. The primers and probes for TaqMan analysis were designed with Primer 3 online software (http://frodo.wi.mit.edu/primer3/; accessed 1 November 2014) based on the sequence information from GenBank. The primers and probes used for heparanase were described in our previous study [20]. The primers and probe for rat podocin (NM_130828) were as follows: 5'-ATC CAG TTC CTG GTG CAA AC-3' (forward primer), 5'- CAC TGA GTC CAA GGC AAC CT-3' (reverse primer), and 5'-
FAM- CAT GAA GCG CCT CTT GGC ACA TC-TAMRA-3' (TaqMan probe). Expression was normalized to glyceraldehyde-3-phosphate dehydrogenase expression in the sample.

\section{Statistical analysis}

Values are expressed as mean \pm SEM. Intergroup differences were evaluated using the Mann-Whitney's U test or Kruskal-Wallis test as appropriate. Post-hoc analysis was performed using Dunnett's test. A P value less than 0.05 was considered statistically significant.

\section{Results}

\section{Cardiovascular and hemodynamic responses of diabetic rats following acute treatment}

Changes in systolic blood pressure (SBP) following acute administration of amlodipine, cilnidipine, or irbesartan are shown in Table 1. SBP significantly decreased following a single administration of amlodipine, cilnidipine, or irbesartan. Combination therapy with irbesartan also significantly reduced SBP and the magnitudes of the reductions ( $\triangle \mathrm{SBP}$ values) were greater than induced by monotherapy, although the differences in $\triangle \mathrm{SBP}$ did not reach statistical significance.

\section{Changes in renal function and glomerular microcirculation of diabetic rats following acute treatment}

Ccr was higher in the DM group than in the Cont group (Table 1). Acute administration of cilnidipine but not amlodipine reduced Ccr. Both irbesartan monotherapy and combination therapy also decreased Ccr (Table 1). Glomerular images were obtained with CCDV probe (Figure 2A). The mean basal diameter of afferent arterioles was greater in the DM group than in the Cont group, while the mean basal diameter of efferent arterioles was lower in the DM group than in the Cont group (Figure 2B). The mean diameter of the afferent arterioles increased 30 min after administration of amlodipine or cilnidipine as monotherapy (Figure 2B). Efferent arterioles also dilated in response to cilnidipine, but amlodipine monotherapy had no effect on efferent arteriole diameter. Irbesartan caused both afferent and efferent arterioles to dilate, not only when administered as monotherapy, but also when administered in combination with cilnidipine or amlodipine. These findings suggest that the intraglomerular pressure may decrease much more by cilnidipine monotherapy than by amlodipine monotherapy, but combination therapy with irbesartan would minimize any difference between the effects of cilnidipine and amlodipine.

\section{Physiological and biochemical changes following chronic treatment}

As shown in Table 2, no significant change in SBP was observed between the DM group and Cont group. Monotherapy with amlodipine or cilnidipine significantly reduced SBP compared with both the

\begin{tabular}{|c|c|c|c|c|c|c|c|}
\hline & Cont & DM & Amlo & Cilni & Irbe & Irbe + Amlo & Irbe + Cilni \\
\hline Number & 4 & 4 & 4 & 4 & 4 & 4 & 4 \\
\hline SBP-Pre & $97 \pm 3$ & $102 \pm 3$ & $102 \pm 5$ & $106 \pm 5$ & $103 \pm 3$ & $105 \pm 3$ & $104 \pm 4$ \\
\hline -Post & $94 \pm 4$ & $100 \pm 3$ & $93 \pm 3^{a}$ & $95 \pm 2^{\mathrm{a}}$ & $94 \pm 4^{\mathrm{a}}$ & $88 \pm 6^{a}$ & $85 \pm 3^{a}$ \\
\hline Ccr & $0.52 \pm 0.05$ & $0.68 \pm 0.08^{b}$ & $0.71 \pm 0.10^{b}$ & $0.62 \pm 0.09$ & $0.56 \pm 0.05$ & $0.59 \pm 0.07$ & $0.53 \pm 0.10$ \\
\hline
\end{tabular}

Data are expressed as mean \pm SEM. ${ }^{a} \mathrm{P}<0.05$ vs. Pre. ${ }^{\mathrm{b}} \mathrm{P}<0.05$ vs. Cont.

SBP: systolic blood pressure (mmHg); Ccr: creatinine clearance (ml/min-BW $100 \mathrm{~g})$; Cont: non-DM control; DM: diabetic control; Amlo: amlodipine; Cilni: cilnidipine; Irbe: irbesartan. 
Citation: Satoh M, Nishi Y, Kadoya H, Itano S, Komai N, et al. (2015) Combination Irbesartan/Amlodipine versus Irbesartan/Cilnidipine for Attenuation of Albuminuria in Rats with Streptozotocin-Induced Diabetic Nephropathy. Pharm Anal Acta 6: 369. doi:10.4172/21532435.1000369

Page 4 of 7

control and DM groups. Combination therapies (Irbe + Amlo or Irbe + Cilni) also decreased SBP, and the final values were significantly lower than in the each monotherapy groups.

Body weight and serum creatinine were significantly higher in the control group compared to all DM model groups (treated or untreated). Serum glucose and blood urea nitrogen were significantly lower in the control group compared to all DM model groups (treated or untreated). There was no significant difference in body weight, serum glucose, serum ctreatinine or blood urea nitrogen between the DM and combination therapy groups.

\section{Drug effects on urinary albumin excretion}

Changes in urinary albumin excretion are shown in Figure 3. Urinary albumin excretion was significantly higher in the DM group at 6 weeks after streptozotocin injection than in the Cont group. After 2 weeks of monotherapy, urinary albumin excretion was significantly reduced by cilnidipine treatment, while amlodipine did not significantly reduce urinary albumin excretion. After 2 weeks of monotherapy with irbesartan $(8 \mathrm{~W}$ Irbe + Amlo or $8 \mathrm{~W}$ Irbe + Cilni groups), urinary albumin excretion was also significantly reduced. The addition of amlodipine or cilnidipine to the irbesartan therapy (10W Irbe + Amlo or 10W Irbe + Cilni) did not induce any further reduction in urinary albumin excretion. Thus, combination therapy of cilnidipine with irbesartan may be equally effective as amlodipine with irbesartan for treating $\mathrm{DN}$.

\section{Evaluation of glomerular oxidative status}

There was no significant difference in glomerular architecture among the Cont and DM groups, with the exception of greatly increased glomerular size in the DM group (Figure 4A, B). Glomerular oxidative status was evaluated by measuring the increase in fluorescence emission
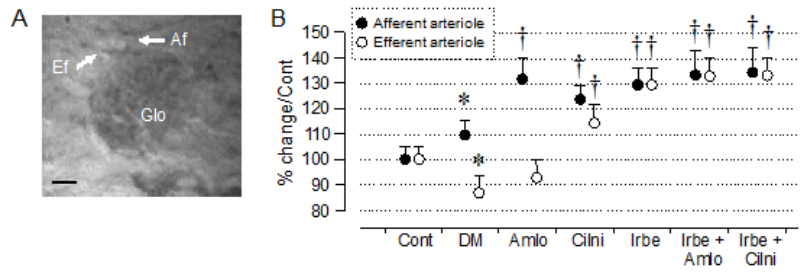

Figure 2: Comparison of glomerular afferent and efferent arteriole diameters before and after acute drug administration in diabetic rats. Typical images of glomeruli in the Cont group (A) recorded using a CCDV microscope. Glo, glomerulus; Af, afferent artery; Ef, efferent artery. Scale bar=50 $\mu \mathrm{m}$. (B) Percent changes in afferent and efferent arteriolar diameters following acute drug treatment compared with the non-diabetic control group. $\mathrm{N}=4$ rats/ group. ${ }^{*} p<0.05$ vs. Cont. ${ }^{\dagger} p<0.05$ vs. DM. associated with the oxidation of DCFH to DCF (Figure 4C, D). At the end of the study, DCF fluorescence intensity in isolated glomeruli was significantly stronger in the DM group than in the Cont group (Figure 4C). All drug treatment regimens except amlodipine monotherapy (Cilni, Irbe + Amlo, and Irbe + Cilni) significantly reduced oxidative stress as measured by DCF fluorescence intensity in the isolated glomeruli.

\section{Effect of combination therapy on endothelial surface layer and heparanase expression}

To further elucidate the mechanisms responsible for suppressing albuminuria in diabetic rats, we evaluated the endothelial surface layer (ESL) and expression of heparanase mRNA in the glomeruli. The glomerular ESL was assessed using WGA staining (Figure 5A). The ESL in glomeruli from control rats had a typical regular appearance and low mean WGA staining score, indicative of a relatively uniform endothelial cell distribution (Figure 5B). Glomeruli in the DM and Amlo groups exhibited markedly degenerated ESL, with higher mean WGA staining scores indicative of variable/discontinuous cell distributions. In contrast, cilnidipine monotherapy and combination therapy with irbesartan plus amlodipine or cilnidipine prevented the deterioration of the ESL (Figure 5B). Heparanase degrades heparan sulphate glycosaminoglycans, the main components of the ESL in glomerular capillaries. Glomeruli from untreated DM rats exhibited a significant increase in heparanase mRNA expression compared to controls (Figure 5C), consistent with the more extensive ESL degeneration. Both cilnidipine monotherapy and combination therapy at least partially reversed this increase in heparanase expression (with no significant difference in expression between these groups). In contrast, amlodipine monotherapy did not alleviate the increased heparanase mRNA expression, possibly accounting for ESL degeneration under this treatment condition.

\section{Effect of combination therapy on podocin expression}

The expression of podocin, a glomerular slit diagram marker, was evaluated using immunohistochemistry and quantitative realtime PCR .As shown in, control glomeruli exhibited robust epithelial podocin staining. In contrast, the glomeruli of untreated diabetic rats and amlodipine-treated rats showed markedly attenuated staining. This loss of glomerular podocin staining was at least partially reversed by cilnidipine monotherapy and by combination treatment with irbesartan $(\mathrm{p}<0.01)$. Likewise, the reduced podocin mRNA expression observed in the DM group was not significantly reversed by amlodipine monotherapy but was partially reversed by cilnidipine monotherapy and combination therapy with irbesartan.

\section{Discussion}

Acute administration of cilnidipine reduced glomerular

\begin{tabular}{|l|l|l|l|l|l|l|}
\hline & Cont & DM & Amlo & Cilni & Irbe + Amlo & 7 \\
\hline Number & 7 & 7 & 7 & 7 & 7 & $98 \pm 3^{\mathrm{a}, \mathrm{b}, \mathrm{c}}$ \\
\hline SBP & $123 \pm 2$ & $126 \pm 2$ & $113 \pm 3^{\mathrm{a}, \mathrm{b}}$ & $111 \pm 3^{\mathrm{a}, \mathrm{b}}$ & $99 \pm 4^{\mathrm{a}, \mathrm{b}, \mathrm{d}}$ \\
\hline BW & $543 \pm 20$ & $382 \pm 13^{\mathrm{a}}$ & $378 \pm 13^{\mathrm{a}}$ & $381 \pm 24^{\mathrm{a}}$ & $401 \pm 18^{\mathrm{a}}$ \\
\hline Serum glucose & $143 \pm 10$ & $623 \pm 31^{\mathrm{a}}$ & $633 \pm 21^{\mathrm{a}}$ & $611 \pm 45^{\mathrm{a}}$ & $594 \pm 23^{\mathrm{a}}$ \\
\hline Serum Cre & $0.21 \pm 0.01$ & $0.16 \pm 0.01^{\mathrm{a}}$ & $0.17 \pm 0.01^{\mathrm{a}}$ & $0.17 \pm 0.01^{\mathrm{a}}$ & $0.16 \pm 0.01^{\mathrm{a}}$ & $6.17 \pm 0.02^{\mathrm{a}}$ \\
\hline BUN & $21.6 \pm 0.8$ & $27.0 \pm 2.4^{\mathrm{a}}$ & $26.8 \pm 1.2^{\mathrm{a}}$ & $28.2 \pm 1.5^{\mathrm{a}}$ & $25.6 \pm 1.4^{\mathrm{a}}$ & $28.4 \pm 2.4^{\mathrm{a}}$ \\
\hline
\end{tabular}

Data are expressed as mean \pm SEM. ${ }^{a} \mathrm{P}<0.05$ vs. Cont. ${ }^{\mathrm{b}}<0.05$ vs. $\mathrm{DM}$. ${ }^{\mathrm{P}}<0.05$ vs. Amlo. ${ }^{\mathrm{d}} \mathrm{P}<0.05$ vs. Cilni.

SBP: systolic blood pressure $(\mathrm{mmHg})$; BW: body weight $(\mathrm{g})$; Cre: Creatinine (mg/dL); BUN: blood urea nitrogen (mg/dL); Cont: non diabetic control; DM: streptozotocininduced diabetes; Amlo: amlodipine; Cilni: cilnidipine; Irbe: irbesartan.

Table 2: Physiological and biochemical changes following chronic treatment. 


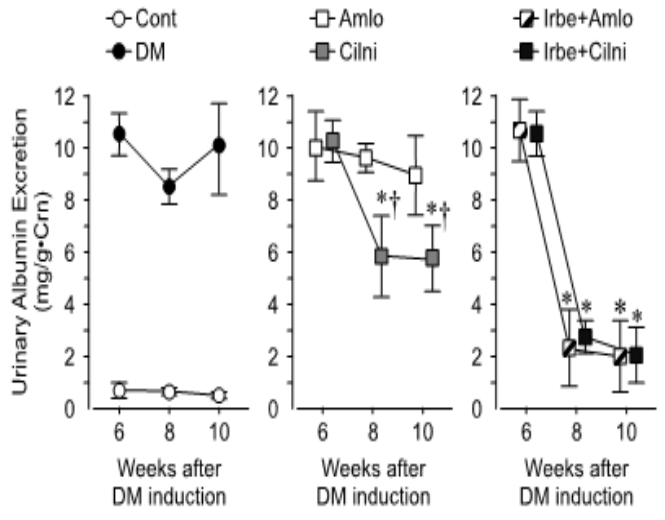

Figure 3: Comparison of urinary albumin excretion rates during chronic drug monotherapy versus combination therapy in diabetic rats. Urinary albumin excretion at 6,8 , and 10 weeks after DM induction, $\mathrm{n}=7$ rats/group. * $p<0.05$ vs. $6 \mathrm{~W}^{\dagger} p<0.05$ vs. Amlo.
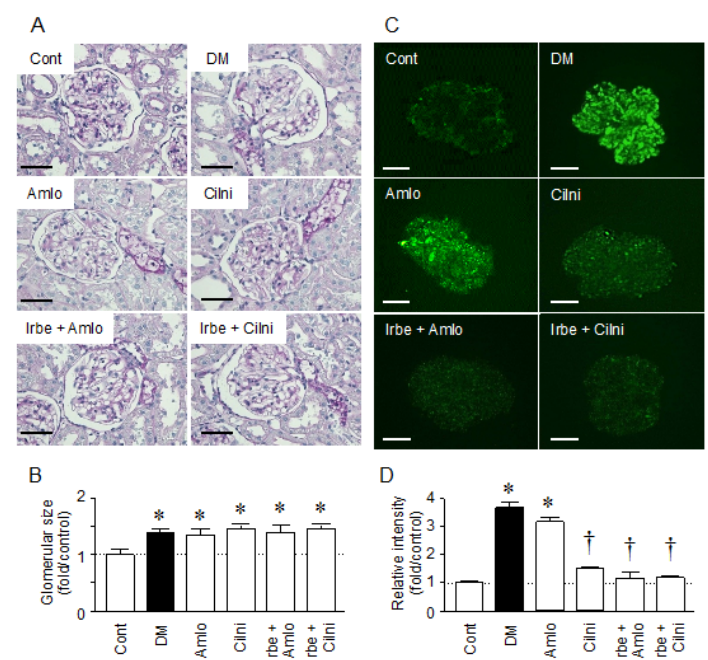

Figure 4: Morphological changes and oxidative status of glomeruli following chronic drug monotherapy and combination therapy. (A) PAS staining. Scale bar=50 $\mu \mathrm{m}$. (B) Glomerular size. ${ }^{*} p<0.05$ vs. Cont. (C) ROS production as bar $=50 \mu \mathrm{m}$. (B) Glomerular size. ${ }^{*} p<0.05$ vs. Cont. (C) ROS production as
measured by DCFH staining in isolated glomeruli. Scale bar $=50 \mu \mathrm{m}$. (D) Relative DCF intensity in glomeruli. $\mathrm{N}=20$ glomeruli for each treatment group. ${ }^{*} p<0.05$ vs. Cont. ${ }^{\dagger} p<0.05$ vs. DM.

hyperfiltration rate more effectively than amlodipine. Moreover, daily administration of cilnidipine suppressed albuminuria associated with $\mathrm{DN}$ to a much greater extent than amlodipine, likely by protecting glomeruli against oxidative stress and ensuing degeneration of the ESL. However, amlodipine was equally effective when administered with irbesartan in both the acute and chronic treatment protocols. Our findings suggest that the addition of irbesartan improves the efficacy of amlodipine to the level of cilnidipine monotherapy, likely by RAS blockage effects that complement the actions of amlodipine.

Cilnidipine, an L/N-type CCB, had a more pronounced vasodilatory effect on glomerular efferent arterioles than amlodipine, an L-type CCB. Furthermore, cilnidipine dilated both afferent and efferent arterioles while amlodipine dilated only the afferent arterioles. Hence, L-type CCBs increase glomerular capillary pressure whereas $\mathrm{L} / \mathrm{N}$-type CCBs alleviate glomerular hypertension [8]. In hypertensive
A

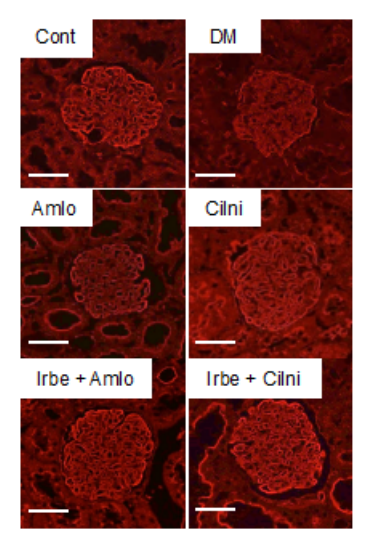

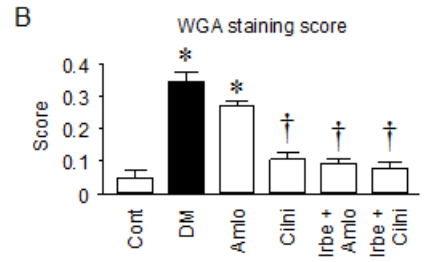

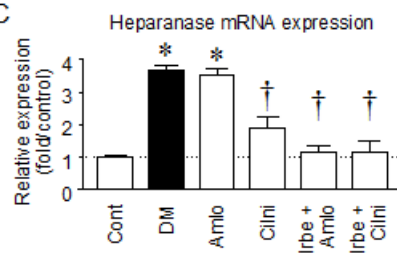

Figure 5: Evaluation of glomerular endothelial surface layer integrity following chronic drug monotherapy or combination therapy. (A) WGA staining. Scale bar $=50 \mu \mathrm{m}$. (B) WGA staining score. $N>80$ tissue samples for each treatment group. ${ }^{\star} p<0.05$ vs. Cont. ${ }^{\dagger} p<0.05$ vs. DM. (C) Relative heparanase mRNA expression in isolated glomeruli. ${ }^{*} p<0.05$ vs. Cont. ${ }^{\dagger} p<0.05$ vs. DM.

patients with renal disease or diabetes, cilnidipine treatment attenuated proteinuria and led to a greater reduction in glomerular filtration rate than amlodipine $[25,26]$. However, we found that combination therapy with irbesartan resulted in a greater reduction in albuminuria compared to either $\mathrm{CCB}$ alone, with equal effectiveness of both combinations. Therefore, irbesartan may be useful for treating DN, particularly in combination with amlodipine or cilnidipine.

The efficacy of combination therapy including the ARB irbesartan for efferent arteriolar vasodilation and albuminuria reduction may be due to the additional attenuation of the RAS. As demonstrated in a previous study, a high dose of cilnidipine or amlodipine produced similar effects on SBP [27]. However, cilnidipine had no effect on plasma renin activity or plasma angiotensin II level, while amlodipine significantly increased these parameters compared to a vehicle group [27]. Hence, irbesartan may counteract RAS activation by amlodipine. Dr. Flynn et al. reported that long-term high dose of amlodipine treatment for streptozotocin-induced diabetic kidney exacerbated albuminuria and increased blood pressure due to increases in renal renin activity [28]. So, combination therapy with ARB may be reasonable for block the adverse effects of amlodipine in normotensive diabetic kidney disease.

In a study of hypertensive model rats, cilnidipine was more effective than amlodipine for preventing renal injury when used in combination with the ARB valsartan [29]. In our study, we used irbesartan instead because it has been shown to effectively treat both early- and late-stage DN [11]. A meta-analysis of short-term, double-blind, parallel group, randomized controlled trials for the treatment of adult hypertension did find evidence supporting the use of valsartan $160 \mathrm{mg}$ for reducing SBP and DBP compared to valsartan $80 \mathrm{mg}$ and irbesartan $150 \mathrm{mg}$ [30], but irbesartan $150 \mathrm{mg}$ had a slower decay and longer duration of antagonistic action than valsartan $80 \mathrm{mg}$ with [12] or without hydrochlorothiazide $[13,14]$. These pharmacologic differences may explain the similar effects of cilnidipine and amlodipine when used in combination with irbesartan compared to valsartan.

Cilnidipine has been shown to inhibit excessive release of norepinephrine from sympathetic nerve endings, including those in 
Citation: Satoh M, Nishi Y, Kadoya H, Itano S, Komai N, et al. (2015) Combination Irbesartan/Amlodipine versus Irbesartan/Cilnidipine for Attenuation of Albuminuria in Rats with Streptozotocin-Induced Diabetic Nephropathy. Pharm Anal Acta 6: 369. doi:10.4172/21532435.1000369

Page 6 of 7

the kidney, and therefore alters neural control of renal function [31]. Thus, cilnidipine has a sympatho-inhibitory effect in patients with hypertension [32], a property not shared by amlodipine. However, irbesartan also displays sympatho-inhibitory potency [33]. In hypertensive patients, treatment with irbesartan for 4 weeks restored sympathetic vasoconstriction [15]. Thus, irbesartan plus amlodipine may possess a similar pharmacological spectrum as irbesartan plus cilnidipine. Recently, irbesartan was identified as a ligand for PPAR $\gamma$ [16] and an inducer of adiponectin [34], an anti-inflammatory that may help prevent DN. Irbesartan has also been reported to normalize the expression of nephrin and to decrease urinary albumin excretion in hypertensive diabetic rats [35]. These pleiotropic effects may complement the actions of amlodipine, thereby abrogating the superior efficacy of cilnidipine.

Irbesartan alone completely inhibited urinary albumin excretion by 8 weeks after DM induction. However, albuminuria was not suppressed any further with added amlodipine or cilnidipine, suggesting that irbesartan monotherapy may be sufficient to prevent the progression of experimental DN in normotensive rats. Alternatively, SBP was decreased to a greater extent by both combination therapies than by either CCB monotherapy. In the management of hypertension, combination therapy with two or more agents is more effective for achieving strict blood pressure control [5,6]. Indeed, combination therapy with irbesartan and amlodipine or cilnidipine is recommended over irbesartan monotherapy.

In conclusion, cilnidipine monotherapy suppressed albuminuria associated with experimental DN more effectively than amlodipine monotherapy. However, combination therapy with irbesartan led to a greater reduction in albuminuria in both treatment groups compared to CCB monotherapy and mitigated the superior effect of cilnidipine. Combination therapy with irbesartan for DN may confer a renoprotective effect beyond that achieved with CCBs alone.

\section{Acknowledgement}

We are grateful to Ms. Etsuko Yorimasa of Kawasaki Medical School for excellent technical assistance.

\section{Financial Support}

This work was supported in part by the Ministry of Health, Labour and Welfare of Japan. N. Komai (No. 23591209) and N. Kashihara (No. 24390218) are recipients of Grants-in-Aid for Scientific Research from the Japan Society for the Promotion of Science. This work was also supported by Research grants from Sumitomo Dainippon Pharma Co. (Osaka, Japan) through a contract with N. Kashihara.

\section{References}

1. Ravera M, Re M, Deferrari L, Vettoretti S, Deferrari G (2006) Importance of blood pressure control in chronic kidney disease. J Am Soc Nephrol 17: S98103.

2. Basile JN (2003) The Importance of Systolic Blood Pressure Control and Cardiovascular Disease Prevention. Curr Treat Options Cardiovasc Med 5 : 271-277.

3. Brewster UC, Setaro JF, Perazella MA (2003) The renin-angiotensinaldosterone system: cardiorenal effects and implications for renal and cardiovascular disease states. Am J Med Sci 326: 15-24.

4. Oparil S, Silfani TN, Walker JF (2005) Role of angiotensin receptor blockers as monotherapy in reaching blood pressure goals. Am J Hypertens 18: 287-294.

5. James PA, Oparil S, Carter BL, Cushman WC, Dennison-Himmelfarb C, et al. (2014) 2014 evidence-based guideline for the management of high blood pressure in adults: report from the panel members appointed to the Eighth Joint National Committee (JNC 8). JAMA 311: 507-520.

6. Shimamoto K, Ando K, Fujita T, Hasebe N, Higaki J, et al. (2014) The Japanese Society of Hypertension Guidelines for the Management of Hypertension (JSH 2014). Hypertens Res 37: 253-390.
7. Aota $Y$, Morimoto S, Sakuma T, Morita T, Jo F, et al. (2009) Efficacy of an Iand $n$-type calcium channel blocker in hypertensive patients with neurovascula compression of the rostral ventrolateral medulla. Hypertens Res 32: 700-705.

8. Hayashi K, Homma K, Wakino S, Tokuyama H, Sugano N, et al. (2010) T-type Ca channel blockade as a determinant of kidney protection. Keio J Med 59: 84-95

9. Miwa Y, Tsuchihashi T, Ohta Y, Tominaga M, Kawano Y, et al. (2010) Antiproteinuric effect of cilnidipine in hypertensive Japanese treated with reninangiotensin-system inhibitors - a multicenter, open, randomized trial using 24hour urine collection. Clin Exp Hypertens 32: 400-405.

10. Schmieder RE, Ruilope LM, BarnettAH (2011) Renal protection with angiotensin receptor blockers: where do we stand. J Nephrol 24: 569-580.

11. Bramlage P, Durand-Zaleski I, Desai N, Pirk O, Hacker C (2009) The value of irbesartan in the management of hypertension. Expert Opin Pharmacother 10: 1817-1831.

12. Bobrie G, Delonca J, Moulin C, Giacomino A, Postel-Vinay N, et al. (2005) A home blood pressure monitoring study comparing the antihypertensive efficacy of two angiotensin II receptor antagonist fixed combinations. Am J Hypertens 18: $1482-1488$

13. Mancia G, Korlipara K, van Rossum P, Villa G, Silvert B (2002) An ambulatory blood pressure monitoring study of the comparative antihypertensive efficacy of two angiotensin II receptor antagonists, irbesartan and valsartan. Blood Press Monit 7: 135-142.

14. Belz GG, Butzer R, Kober S, Mang C, Mutschler E (1999) Time course and extent of angiotensin II antagonism after irbesartan, losartan, and valsartan in humans assessed by angiotensin II dose response and radioligand receptor assay. Clin Pharmacol Ther 66: 367-373.

15. Vongpatanasin W, Wang Z, Arbique D, Arbique G, Adams-Huet B, et al. (2011) Functional sympatholysis is impaired in hypertensive humans. J Physiol 589 : 1209-1220.

16. Iwai M, Kanno H, Senba I, Nakaoka H, Moritani T, et al. (2011) Irbesartan increased PPAR $\hat{I}^{3}$ activity in vivo in white adipose tissue of atherosclerotic mice and improved adipose tissue dysfunction. Biochem Biophys Res Commun 406 123-126.

17. Satoh M, Fujimoto S, Arakawa S, Yada T, Namikoshi T, et al. (2008) Angiotensin II type 1 receptor blocker ameliorates uncoupled endothelial nitric oxide synthase in rats with experimental diabetic nephropathy. Nephrol Dial Transplant 23: 3806-3813.

18. Nagasu H, Satoh M, Fujimoto S, Tomita N, Sasaki T, et al. (2012) Azelnidipine attenuates glomerular damage in Dahl salt-sensitive rats by suppressing sympathetic nerve activity. Hypertens Res 35: 348-355.

19. Fujimoto S, Satoh M, Nagasu H, Horike H, Sasaki T, et al. (2009) Azelnidipine exerts renoprotective effects by improvement of renal microcirculation in angiotensin II infusion rats. Nephrol Dial Transplant 24: 3651-3658.

20. Kuwabara A, Satoh M, Tomita N, Sasaki T, Kashihara N (2010) Deterioration of glomerular endothelial surface layer induced by oxidative stress is implicated in altered permeability of macromolecules in Zucker fatty rats. Diabetologia 53 2056-2065.

21. Pagtalunan ME, Drachman JA, Meyer TW (2000) Methods for estimating the volume of individual glomeruli. Kidney Int 57: 2644-2649.

22. Macconi D, Ghilardi M, Bonassi ME, Mohamed El, Abbate M, et al (2000) Effect of angiotensin-converting enzyme inhibition on glomerular basement membrane permeability and distribution of zonula occludens- 1 in MWF rats. $J$ Am Soc Nephrol 11: 477-489.

23. Fujimoto $S$, Satoh $M$, Horike $H$, Hatta $H$, Haruna $Y$, et al. (2008) Olmesartan ameliorates progressive glomerular injury in subtotal nephrectomized rats through suppression of superoxide production. Hypertens Res 31: 305-313.

24. Kobayashi S, Satoh M, Namikoshi T, Haruna Y, Fujimoto S, et al. (2008) Blockade of serotonin $2 \mathrm{~A}$ receptor improves glomerular endothelial function in rats with streptozotocin-induced diabetic nephropathy. Clin Exp Nephrol 12 119-125

25. Kojima S, Shida M, Yokoyama H (2004) Comparison between cilnidipine and amlodipine besilate with respect to proteinuria in hypertensive patients with renal diseases. Hypertens Res 27: 379-385.

26. Masuda T, Ogura MN, Moriya T, Takahira N, Matsumoto T, et al. (2011) 
Citation: Satoh M, Nishi Y, Kadoya H, Itano S, Komai N, et al. (2015)Combination Irbesartan/Amlodipine versus Irbesartan/Cilnidipine for Attenuation of Albuminuria in Rats with Streptozotocin-Induced Diabetic Nephropathy. Pharm Anal Acta 6: 369. doi:10.4172/21532435.1000369

Beneficial effects of $\mathrm{L}$ - and $\mathrm{N}$-type calcium channel blocker on glucose and lipid metabolism and renal function in patients with hypertension and type II diabetes mellitus. Cardiovasc Ther 29: 46-53.

27. Konda T, Enomoto A, Aritomi S, Niinuma K, Koganei H, et al. (2009) Different effects of L/N-type and L-type calcium channel blockers on the reninangiotensin-aldosterone system in SHR/Izm. Am J Nephrol 30: 155-161.

28. Flynn E, Marbury DC, Sawyer RT, Lee J, Teutsch C, et al. (2012) Amlodipine Reduces Inflammation despite Promoting Albuminuria in the StreptozotocinInduced Diabetic Rat. Nephron Extra 2: 205-218.

29. Aritomi S, Koganei H, Wagatsuma H, Mitsui A, Ogawa T, et al. (2010) The $\mathrm{N}$-type and L-type calcium channel blocker cilnidipine suppresses renal injury in Dahl rats fed a high-salt diet. Heart Vessels 25: 549-555.

30. Nixon RM, Müller E, Lowy A, Falvey H (2009) Valsartan vs. other angiotensin I receptor blockers in the treatment of hypertension: a meta-analytical approach. Int J Clin Pract 63: 766-775
31. Takahara A, Dohmoto $H$, Hisa H, Satoh S, Yoshimoto $R$ (1997) Cilnidipine attenuates renal nerve stimulation-induced renal vasoconstriction and antinatriuresis in anesthetized dogs. Jpn J Pharmacol 75: 27-32.

32. Kishi T, Hirooka Y, Konno S, Sunagawa K (2009) Cilnidipine inhibits the sympathetic nerve activity and improves baroreflex sensitivity in patients with hypertension. Clin Exp Hypertens 31: 241-249.

33. Balt J, Mathy MJ, Pfaffendorf M, van Zwieten PA (2003) Sympatho-inhibitory actions of irbesartan in pithed spontaneously hypertensive and Wistar-Kyoto rats. Fundam Clin Pharmacol 17: 83-91.

34. Clasen R, Schupp M, Foryst-Ludwig A, Sprang C, Clemenz M, et al. (2005) PPARgamma-activating angiotensin type-1 receptor blockers induce adiponectin. Hypertension 46: 137-143.

35. Bonnet F, Cooper ME, Kawachi H, Allen TJ, Boner G, et al (2001) Irbesartan normalises the deficiency in glomerular nephrin expression in a model of diabetes and hypertension. Diabetologia 44: 874-877. 